\title{
Synthesis and biological activities of some new spiro 1,2,4-triazole derivatives having sulfonamide moiety
}

\author{
Hany M. Dalloul $\odot^{1 *}$, Khaled El-nwairy $\odot^{2}$, Ali Z. Shorafa $\odot^{2}$ and \\ Ahmed Abu Samaha ${ }^{3}$ \\ ${ }^{1}$ Department of Chemistry, Faculty of Applied Science, Alaqsa University of Gaza, P.O.Box 4051, Gaza \\ 76888 , Palestine \\ ${ }^{2}$ Ministry of Education \& Higher Education, Beirut Street, Southern Reemal, \\ Gaza 76888, Palestine \\ ${ }^{3}$ Department of Biology, Faculty of Applied Science, Alaqsa University of Gaza, P.O.Box 4051, Gaza \\ 76888, Palestine
}

(Received August 25, 2017; Revised September 09, 2017; Accepted September 11, 2017)

\begin{abstract}
A new series of spiro 1,2,4-triazoles 5-9a-j were prepared by the reaction of appropriate amidrazones 4 with cyclic ketones in catalytic amount of $p$-toluene sulfonic acid. The structures of the titled compounds have been elucidated by the elemental analysis and spectroscopic data (IR, ${ }^{1} \mathrm{H}$ NMR, ${ }^{13} \mathrm{C}$ NMR and MS). The biological activities of the prepared compounds were investigated using well-established methods from the literature.
\end{abstract}

Keywords: Amidrazone; nitrilimines; spiro 1,2,4-triazole; sulfonamide; cyclic ketone. C2017 ACG Publications. All rights reserved.

\section{Introduction}

Amidrazones represent a class of substances with interesting biological properties. The interest in amidrazones and their derivatives stems not only from their biological relevance but also from their applications as precursors and intermediates for the synthesis of many heterocyclic compounds ${ }^{1-3}$ and as ligands in coordination chemistry. It has been established that they can exhibit antibacterial, antifungal ${ }^{4-6}$, antitumor $^{7}$ and antituberculosis activities ${ }^{8}$. They were also found as effective herbicides ${ }^{9}$, pesticides ${ }^{10}$, and insecticides ${ }^{11}$. Amidrazones synthesized from different hydrazonoyl halides were reported to react with $\alpha$ haloesters in the presence of triethylamine as a base under reflux to afford 1,3,5-substituted 4,5-dihydro1,2,4-triazin-6-ones ${ }^{12}$. Several studies, reported the formation and investigation of biological activities of some spiroheterocyclic compounds having triazole, tetrazine thiadiazole, thiadiazines, thiazolidinone and triazine moieties ${ }^{13-17}$. Recently, unknown dispiroheterocycles containing triazole and tetrazine moieties have been disclosed ${ }^{14-17}$.

Sulfonamide derivatives are known to exhibit various pharmacological properties such as antiangiogenic, anti-tumor, anti-inflammatory and anti-analgesic, anti-tubercular, anti-glaucoma, anti-HIV, cytotoxic, anti-microbial, anti-malarial agents ${ }^{18}$. Considering the previous reports on the biological activities and syntheses of sulfonamides we report here synthesis and antimicrobial activity of new spiroheterocycles having 1,2,4-triazole and piperidone derivatives via cyclization of amidrazones containing sulfonamide moiety with cyclic ketones.

\footnotetext{
* Corresponding author: E-mail: hmdalloul@yahoo.com 


\section{Experimental}

\subsection{Reagents and Instrumentation}

Melting points were determined on an A. Krüss Melting Point Meter equipped with a thermometer and are uncorrected. The IR spectra were measured as potassium bromide pellets using a Satellite $3000 \mathrm{Mid}$ infrared spectrophotometer. The ${ }^{1} \mathrm{H}$ NMR and ${ }^{13} \mathrm{C}$ NMR spectra were recorded on a Bruker AM $300 \mathrm{MHz}$ spectrometer at room temperature in DMSO- $d_{6}$ solution using tetramethylsilane (TMS) as internal reference. Chemical shifts were recorded as $\delta$ values in parts per millions (ppm) downfield from internal TMS. Electron impact (EI) mass spectra were run on a Shimadzu GCMSQP1000 EX spectrometer at $70 \mathrm{eV}$. All compounds were analyzed satisfactorily for $\mathrm{C}, \mathrm{H}$ and $\mathrm{N}$. The amidrazones 4 were prepared according to the literature procedures ${ }^{19-22}$. Sodium azide, triphenylphosphine and tetrabutylammonium iodide were obtained from Fluka Chemie Company, Switzerland. Sulfathiazole, sulfadiazine, dioxane, tetrahydrofuran (THF) and triethylamine were purchased from Avocado Research Chemicals, England, and used without further purification.

\subsection{Synthesis of Spiro 4,5-dihyro-1,2,4-triazole derivatives (5-9a-j).}

To a stirred solution of the appropriate amidrazone $4(10 \mathrm{mmol})$ and the respective cycloalkanone $(20 \mathrm{mmol})$ in dioxane $(50 \mathrm{~mL})$, the catalytic amount of $p$-toluenesulfonic acid $(0.1 \mathrm{~g})$ was added. The reaction mixture was refluxed to the completion (monitoring the reaction progress by TLC). The excess of the solvent was evaporated and the residue was triturated with methanol or ethanol. The resulting crude solid product was collected and recrystallized from ethanol. The following compounds were prepared using this method:

3-Acetyl-1-[4-(thiazol-2-yl-sulfonyl)phenyl]-1,2,4-triazaspiro[4.4]non-2-ene (5a): Yield: 65\%; m.p.: 197$199{ }^{\circ} \mathrm{C}$; IR (KBr) v $v_{\max }: \mathrm{cm}^{-1} 3382,3355(\mathrm{NH}), 1695\left(\mathrm{CH}_{3}-\mathrm{C}=\mathrm{O}\right), 1622(\mathrm{C}=\mathrm{N}), 1346,1175\left(\mathrm{SO}_{2}\right) ;{ }^{1} \mathrm{H}$ NMR (DMSO- $\left.d_{6}\right): \delta 11.70\left(s, 1 \mathrm{H}, \mathrm{SO}_{2} \mathrm{NH}\right), 8.27-7.06(m, 4 \mathrm{H}, \mathrm{Ar}-\mathrm{H}), 8.71(\mathrm{~d}, 1 \mathrm{H}$, thiazole), $6.62(\mathrm{~d}, 1 \mathrm{H}$, thiazole), $5.65(\mathrm{~s}, 1 \mathrm{H}, \mathrm{NH}$ triazole ring $), 2.45\left(\mathrm{~s}, 3 \mathrm{H}, \mathrm{COCH}_{3}\right), 2.37-1.60(\mathrm{~m}, 8 \mathrm{H}$, cyclopentane $) ;{ }^{13} \mathrm{C}$ NMR (DMSO- $\left.d_{6}\right): \delta 192.3(\mathrm{C}=\mathrm{O}), 169.1,142.2,139.6,135.4,129.6,127.4,119.1,116.5(\mathrm{Ar}-\mathrm{C}, \mathrm{C}=\mathrm{N}$ and thiazole-C), 92.8 (spiro-C), 34.9, 24.9 (cyclopentane-C), $26.5\left(\mathrm{CH}_{3}\right)$; MS: $\mathrm{m} / z 405\left[\mathrm{M}^{+}\right]$; Anal. Calcd. for $\mathrm{C}_{17} \mathrm{H}_{19} \mathrm{~N}_{5} \mathrm{O}_{3} \mathrm{~S}_{2}$ (405.50): C, 50.35; H, 4.72; N, 17.27; Found: C, 50.57; H, 4.60; N, 17.15.

3-Acetyl-1-[4-(pyrimidin-2-yl-sulfonyl)phenyl]-1,2,4-triazaspiro[4.4]non-2-ene (5b): Yield: 71\%; m.p.: 216-218 ${ }^{\circ} \mathrm{C}$; IR (KBr) $v_{\max }: \mathrm{cm}^{-1} 3374,3350(\mathrm{NH}), 1693\left(\mathrm{CH}_{3}-\mathrm{C}=\mathrm{O}\right), 1626(\mathrm{C}=\mathrm{N}), 1346,1175\left(\mathrm{SO}_{2}\right) ;{ }^{1} \mathrm{H}$ NMR (DMSO-d $\left.)_{6}\right): \delta 11.74\left(s, 1 \mathrm{H}, \mathrm{SO}_{2} \mathrm{NH}\right), 8.27-7.06(m, 4 \mathrm{H}, \mathrm{Ar}-\mathrm{H}), 8.41(\mathrm{~d}, 2 \mathrm{H}$, pyrimidine), $6.83(\mathrm{t}$, $1 \mathrm{H}$, pyrimidine), $5.63\left(\mathrm{~s}, 1 \mathrm{H}, \mathrm{NH}\right.$ triazole ring), $2.55\left(\mathrm{~s}, 3 \mathrm{H}, \mathrm{COCH}_{3}\right), 2.37-1.60(\mathrm{~m}, 8 \mathrm{H}$, cyclopentane); ${ }^{13} \mathrm{C}$ NMR (DMSO- $\left.d_{6}\right): \delta 192.1(\mathrm{C}=\mathrm{O}), 168.1,156.4,142.2,139.7,129.8,127.3,119.2,110.6(\mathrm{Ar}-\mathrm{C}, \mathrm{C}=\mathrm{N}$ and pyrimidine-C), 92.6 (spiro-C), 34.8, 24.6 (cyclopentane-C), $26.6\left(\mathrm{CH}_{3}\right) ; \mathrm{MS}: \mathrm{m} / z 400\left[\mathrm{M}^{+}\right]$; Anal. Calcd. for $\mathrm{C}_{18} \mathrm{H}_{20} \mathrm{~N}_{6} \mathrm{O}_{3} \mathrm{~S}$ (400.46): C, 53.99; H, 5.03; N, 20.99; Found: C, 54.23; H, 4.92; N, 21.10.

3-Acetyl-1-[4-(thiazol-2-yl-sulfonyl)phenyl]-1,2,4-triazaspiro[4.5]dec-2-ene (5c): Yield: 66\%; m.p.: 204$206{ }^{\circ} \mathrm{C}$; IR (KBr) v $v_{\max }: \mathrm{cm}^{-1}$ 3369, $3357(\mathrm{NH}), 1695\left(\mathrm{CH}_{3}-\mathrm{C}=\mathrm{O}\right), 1624(\mathrm{C}=\mathrm{N}), 1346,1175\left(\mathrm{SO}_{2}\right) ;{ }^{1} \mathrm{H}$ NMR (DMSO- $\left.d_{6}\right): \delta 11.76\left(s, 1 \mathrm{H}, \mathrm{SO}_{2} \mathrm{NH}\right), 8.27-7.06(m, 4 \mathrm{H}, \mathrm{Ar}-\mathrm{H}), 8.71(\mathrm{~d}, 1 \mathrm{H}$, thiazole $), 6.65(\mathrm{~d}, 1 \mathrm{H}$, thiazole $), \quad 5.65(\mathrm{~s}, 1 \mathrm{H}, \mathrm{NH}$ triazole ring $), 2.54\left(\mathrm{~s}, 3 \mathrm{H}, \mathrm{COCH}_{3}\right), 2.07-1.16(\mathrm{~m}, 10 \mathrm{H}$, cyclohexane $) ;{ }^{13} \mathrm{C}$ NMR (DMSO- $\left.d_{6}\right): \delta 192.4(\mathrm{C}=\mathrm{O}), 168.4,142.2,139.3,135.5,129.7,127.3,119.2,116.2(\mathrm{Ar}-\mathrm{C}, \mathrm{C}=\mathrm{N}$ and thiazole-C), 92.1 (spiro-C), 35.9, 24.8, 23.2 (cyclohexane-C), $26.5\left(\mathrm{CH}_{3}\right) ; \mathrm{MS}: \mathrm{m} / z 419\left[\mathrm{M}^{+}\right]$; Anal. Calcd. for $\mathrm{C}_{18} \mathrm{H}_{21} \mathrm{~N}_{5} \mathrm{O}_{3} \mathrm{~S}_{2}$ (419.53): C, 61.53; H, 5.05; N, 16.69; Found: C, 61.70; H, 4.96; N, 16.55 .

3-Acetyl-1-[4-(pyrimidin-2-yl-sulfonyl)phenyl]-1,2,4-triazaspiro[4.5]dec-2-ene (5d): Yield: 65\%; m.p.: 192-194 ${ }^{\circ} \mathrm{C}$; IR (KBr) $v_{\max }: \mathrm{cm}^{-1} 3377,3357(\mathrm{NH}), 1693\left(\mathrm{CH}_{3}-\mathrm{C}=\mathrm{O}\right), 1628(\mathrm{C}=\mathrm{N}), 1346,1175\left(\mathrm{SO}_{2}\right) ;{ }^{1} \mathrm{H}$ NMR (DMSO- $\left.d_{6}\right): \delta 11.71\left(s, 1 \mathrm{H}, \mathrm{SO}_{2} \mathrm{NH}\right), 8.27-7.06(m, 4 \mathrm{H}, \mathrm{Ar}-\mathrm{H}), 8.40(\mathrm{~d}, 2 \mathrm{H}$, pyrimidine $), 6.82(\mathrm{t}$, $1 \mathrm{H}$, pyrimidine), $5.61\left(\mathrm{~s}, 1 \mathrm{H}, \mathrm{NH}\right.$ triazole ring), $2.55\left(\mathrm{~s}, 3 \mathrm{H}, \mathrm{COCH}_{3}\right), 1.98-1.16(\mathrm{~m}, 10 \mathrm{H}$, cyclohexane); ${ }^{13} \mathrm{C}$ NMR (DMSO- $\left.d_{6}\right): \delta 191.9(\mathrm{C}=\mathrm{O}), 168.2,156.5,142.3,139.4,135.5,129.6,127.5,119.3,110.4(\mathrm{Ar}-$ $\mathrm{C}, \mathrm{C}=\mathrm{N}$ and pyrimidine-C), 91.8 (spiro-C), 35.8, 24.6, 23.1 (cyclohexane-C), $26.4\left(\mathrm{CH}_{3}\right) ; \mathrm{MS}: \mathrm{m} / z 414$ 
$\left[\mathrm{M}^{+}\right]$; Anal. Calcd. for $\mathrm{C}_{19} \mathrm{H}_{22} \mathrm{~N}_{6} \mathrm{O}_{2} \mathrm{~S}$ (414.49): C, 55.06; H, 5.35; N, 20.28; Found: C, 54.78; H, 5.50; N, 20.39 .

3-Acetyl-1-[4-(thiazol-2-yl-sulfonyl)phenyl]-1,2,4-triazaspiro[4.6]undec-2-ene (5e): Yield: 67\%; m.p.: $178-180{ }^{\circ} \mathrm{C}$; IR ( $\left.\mathrm{KBr}\right) v_{\max }$ : $\mathrm{cm}^{-1} 3375,3358\left(\mathrm{NH}^{\prime} \mathrm{s}\right), 1695\left(\mathrm{CH}_{3}-\mathrm{C}=\mathrm{O}\right), 1625(\mathrm{C}=\mathrm{N}), 1346,1175\left(\mathrm{SO}_{2}\right) ;{ }^{1} \mathrm{H}$ NMR (DMSO- $\left.d_{6}\right): \delta 11.76,(\mathrm{~s}, 1 \mathrm{H}, \mathrm{NH}), 8.27-7.06(m, 4 \mathrm{H}, \mathrm{Ar}-\mathrm{H}), 8.70(\mathrm{~d}, 1 \mathrm{H}$, thiazole $), 6.64(\mathrm{~d}, 1 \mathrm{H}$, thiazole), $5.63(\mathrm{~s}, 1 \mathrm{H}, \mathrm{NH}$ triazole ring $), 2.54\left(\mathrm{~s}, 3 \mathrm{H}, \mathrm{COCH}_{3}\right), 2.35-1.42(\mathrm{~m}, 12 \mathrm{H}$, cycloheptane $) ;{ }^{13} \mathrm{C}$ NMR (DMSO- $\left.d_{6}\right): \delta 192.4(\mathrm{C}=\mathrm{O}), 168.2,142.3,139.4,135.3,129.7,127.4,119.3,116.5(\mathrm{Ar}-\mathrm{C}, \mathrm{C}=\mathrm{N}$ and

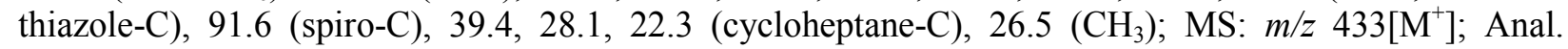
Calcd. for $\mathrm{C}_{19} \mathrm{H}_{23} \mathrm{~N}_{5} \mathrm{O}_{3} \mathrm{~S}_{2}$ (433.55): C, 52.64; H, 5.35; N, 16.15; Found: C, 52.45; H, 5.24; N, 16.03 .

3-Acetyl-1-[4-(pyrimidin-2-yl-sulfonyl)phenyl]-1,2,4-triazaspiro[4.6]undec-2-ene (5f): Yield: 64\%; m.p.: 188-190 ${ }^{\circ} \mathrm{C}$; IR (KBr) $v_{\max }: \mathrm{cm}^{-1} 3373,3365(\mathrm{NH}), 1695\left(\mathrm{CH}_{3}-\mathrm{C}=\mathrm{O}\right), 1620(\mathrm{C}=\mathrm{N}), 1346,1175\left(\mathrm{SO}_{2}\right) ;{ }^{1} \mathrm{H}$ NMR (DMSO- $\left.d_{6}\right): \delta 11.69\left(s, 1 \mathrm{H}, \mathrm{SO}_{2} \mathrm{NH}\right), 8.27-7.06(m, 4 \mathrm{H}, \mathrm{Ar}-\mathrm{H}), 8.36(\mathrm{~d}, 2 \mathrm{H}$, pyrimidine), $6.81(\mathrm{t}$, $1 \mathrm{H}$, pyrimidine), $5.62\left(\mathrm{~s}, 1 \mathrm{H}, \mathrm{NH}\right.$ triazole ring), $2.56\left(\mathrm{~s}, 3 \mathrm{H}, \mathrm{COCH}_{3}\right), 2.34-1.40(\mathrm{~m}, 12 \mathrm{H}$, cycloheptane); ${ }^{13} \mathrm{C}$ NMR (DMSO- $\left.d_{6}\right): \delta 191.8(\mathrm{C}=\mathrm{O}), 168.3,156.7,142.5,139.6,135.1,129.3,127.4,119.2,110.4$ (Ar$\mathrm{C}, \mathrm{C}=\mathrm{N}$ and pyrimidine-C), 39.1, 28.3, 22.5 (cycloheptane-C), 91.8 (spiro-C), $26.4\left(\mathrm{CH}_{3}\right) ; \mathrm{MS}: \mathrm{m} / z 428$ [M $]$; Anal. Calcd. for $\mathrm{C}_{20} \mathrm{H}_{24} \mathrm{~N}_{6} \mathrm{O}_{3} \mathrm{~S}$ (428.52): C, 56.06; H, 5.65; N, 19.61; Found: C, 55.86; H, 5.53; N, 19.47 .

3-Acetyl-1-[4-(thiazol-2-yl-sulfonyl)phenyl]-1,2,4-triazaspiro[4.7]dodec-2-ene (5g): Yield: 70\%; m.p.: 166-168 ${ }^{\circ} \mathrm{C}$; IR ( $\left.\mathrm{KBr}\right) v_{\max }: \mathrm{cm}^{-1} 3373,3361(\mathrm{NH}), 1696\left(\mathrm{CH}_{3} \mathrm{C}=\mathrm{O}\right), 1627(\mathrm{C}=\mathrm{N}), 1346,1175\left(\mathrm{SO}_{2}\right) ;{ }^{1} \mathrm{H}$ NMR (DMSO- $\left.d_{6}\right): \delta 11.71\left(s, 1 \mathrm{H}, \mathrm{SO}_{2} \mathrm{NH}\right), 8.27-7.06(m, 4 \mathrm{H}, \mathrm{Ar}-\mathrm{H}), 8.72(\mathrm{~d}, 1 \mathrm{H}$, thiazole $), 6.61(\mathrm{~d}, 1 \mathrm{H}$, thiazole), $5.65(\mathrm{~s}, 1 \mathrm{H}, \mathrm{NH}$ triazole ring $), 2.55\left(\mathrm{~s}, 3 \mathrm{H}, \mathrm{COCH}_{3}\right), 2.42-1.24(\mathrm{~m}, 14 \mathrm{H}$, cyclooctane $) ;{ }^{13} \mathrm{C}$ NMR (DMSO- $\left.d_{6}\right): \delta 192.3(\mathrm{C}=\mathrm{O}), 168.5,142.3,139.7,135.5,129.4,127.6,119.4,116.5(\mathrm{Ar}-\mathrm{C}, \mathrm{C}=\mathrm{N}$ and thiazole-C), 90.9 (spiro-C), 41.9, 36.5, 27.2, 21.8 (cyclooctane-C), $26.5\left(\mathrm{CH}_{3}\right)$; $\mathrm{MS}: \mathrm{m} / z 447\left[\mathrm{M}^{+}\right]$; Anal. Calcd. for $\mathrm{C}_{20} \mathrm{H}_{25} \mathrm{~N}_{5} \mathrm{O}_{3} \mathrm{~S}_{2}$ (447.58): C, 53.67; H, 5.63; N, 15.65; Found: C, 53.45; H, 5.75; N, 15.55 .

3-Acetyl-1-[4-(pyrimidin-2-yl-sulfonyl)phenyl]-1,2,4-triazaspiro[4.7]dodec-2-ene (5h): Yield: 65\%; m.p.: 169-171 ${ }^{\circ} \mathrm{C}$; IR (KBr) $v_{\max }: \mathrm{cm}^{-1} 3366,3360(\mathrm{NH}), 1692\left(\mathrm{CH}_{3}-\mathrm{C}=\mathrm{O}\right), 1626(\mathrm{C}=\mathrm{N}), 1346,1175\left(\mathrm{SO}_{2}\right) ;{ }^{1} \mathrm{H}$ NMR (DMSO- $\left.d_{6}\right): \delta 11.76\left(s, 1 \mathrm{H}, \mathrm{SO}_{2} \mathrm{NH}\right), 8.27-7.06(m, 4 \mathrm{H}, \mathrm{Ar}-\mathrm{H}), 8.40(\mathrm{~d}, 2 \mathrm{H}$, pyrimidine $), 6.81(\mathrm{t}$, $1 \mathrm{H}$, pyrimidine), $5.66\left(\mathrm{~s}, 1 \mathrm{H}, \mathrm{NH}\right.$ triazole ring), $2.55\left(\mathrm{~s}, 3 \mathrm{H}, \mathrm{COCH}_{3}\right), 2.42-1.24(\mathrm{~m}, 14 \mathrm{H}$, cyclooctane); ${ }^{13} \mathrm{C}$ NMR (DMSO- $\left.d_{6}\right): \delta 192.2(\mathrm{C}=\mathrm{O}), 168.1,156.3,142.0,139.4,135.2,129.4,127.2,119.0,110.2(\mathrm{Ar}-$ $\mathrm{C}, \mathrm{C}=\mathrm{N}$ and pyrimidine-C), 91.8 (spiro-C), 41.8, 36.3, 27.1, 21.6 (cyclooctane-C), $26.6\left(\mathrm{CH}_{3}\right) ; \mathrm{MS}: \mathrm{m} / \mathrm{z}$ $442\left[\mathrm{M}^{+}\right]$; Anal. Calcd. for $\mathrm{C}_{21} \mathrm{H}_{26} \mathrm{~N}_{6} \mathrm{O}_{3} \mathrm{~S}$ (442.54): C, 57.00; H, 5.92; N, 18.99; Found: C, 56.85; H, 6.05; $\mathrm{N}, 18.87$.

3-Acetyl-8-methyl-1-[4-(thiazol-2-yl-sulfonyl)phenyl]-1,2,4-triazaspiro[4.5]dec-2-ene (5i): Yield: 71\%; m.p.: $186-188^{\circ} \mathrm{C}$; IR (KBr) v $v_{\max }: \mathrm{cm}^{-1} 3374,3355(\mathrm{NH}), 1693\left(\mathrm{CH}_{3}-\mathrm{C}=\mathrm{O}\right), 1622(\mathrm{C}=\mathrm{N}), 1346,1175$ $\left(\mathrm{SO}_{2}\right) ;{ }^{1} \mathrm{H}$ NMR (DMSO- $\left.d_{6}\right): \delta 11.70\left(s, 1 \mathrm{H}, \mathrm{SO}_{2} \mathrm{NH}\right), 8.27-7.06(m, 4 \mathrm{H}, \mathrm{Ar}-\mathrm{H}), 8.71(\mathrm{~d}, 1 \mathrm{H}$, thiazole), $6.65(\mathrm{~d}, 1 \mathrm{H}$, thiazole $), 5.64(\mathrm{~s}, 1 \mathrm{H}, \mathrm{NH}$ triazole ring $), 2.54\left(s, 3 \mathrm{H}, \mathrm{COCH}_{3}\right), 2.07-1.14(\mathrm{~m}, 8 \mathrm{H}$, cyclohexane), $0.98\left(3 \mathrm{H}, \mathrm{s}, \mathrm{CH}_{3}\right) ;{ }^{13} \mathrm{C}$ NMR (DMSO- $\left.d_{6}\right): \delta 189.9(\mathrm{C}=\mathrm{O}), 168.2,142.2,139.6,135.4,129.6$, 127.4, 119.2, 116.5 (Ar-C, $\mathrm{C}=\mathrm{N}$ and thiazole-C), 34.9, 27.8, 22.4 (cyclohexane-C), 90.9 (spiro-C), 31.4 $\left(\mathrm{CH}_{3}\right.$ at cyclohexane ring) $26.5\left(\mathrm{CH}_{3}\right)$; MS: $\mathrm{m} / z 433\left[\mathrm{M}^{+}\right]$; Anal. Calcd. for $\mathrm{C}_{19} \mathrm{H}_{23} \mathrm{~N}_{5} \mathrm{O}_{3} \mathrm{~S}_{2}$ (433.55): C, 52.64; H, 5.35; N, 16.15; Found: C, 52.85; H, 5.20; N, 16.28.

3-Acetyl-8-tert-butyl-1-[4-(thiazol-2-yl-sulfonyl)phenyl]-1,2,4-triazaspiro-[4.5]dec-2-ene (5j): Yield: 69\%; m.p.: 199-201 ${ }^{\circ} \mathrm{C}$; IR ( $\left.\mathrm{KBr}\right) v_{\max }: \mathrm{cm}^{-1} 3370,3353$, $(\mathrm{NH}), 1695\left(\mathrm{CH}_{3}-\mathrm{C}=\mathrm{O}\right) 1622(\mathrm{C}=\mathrm{N}), 1346$, $1175\left(\mathrm{SO}_{2}\right) ;{ }^{1} \mathrm{H}$ NMR (DMSO- $\left.d_{6}\right): \delta 11.69\left(s, 1 \mathrm{H}, \mathrm{SO}_{2} \mathrm{NH}\right), 8.27-7.06(m, 4 \mathrm{H}, \mathrm{Ar}-\mathrm{H}), 8.68(\mathrm{~d}, 1 \mathrm{H}$, thiazole), $6.63(\mathrm{~d}, 1 \mathrm{H}$, thiazole $), 5.57(\mathrm{~s}, 1 \mathrm{H}, \mathrm{NH}$ triazole ring $), 2.57\left(\mathrm{~s}, 3 \mathrm{H}, \mathrm{COCH}_{3}\right), 2.05-1.13(\mathrm{~m}, 8 \mathrm{H}$, cyclohexane), $0.92\left(9 \mathrm{H}, \mathrm{s},\left(\mathrm{CH}_{3}\right)_{3} \mathrm{C}\right) ;{ }^{13} \mathrm{C}$ NMR (DMSO- $\left.d_{6}\right): \delta 189.9(\mathrm{C}=\mathrm{O}), 168.2,142.2,139.6,135.4$, 129.6, 127.4, 119.1, 116.5 (Ar-C, $\mathrm{C}=\mathrm{N}$ and thiazole-C), 90.8 (spiro-C), 35.2, 27.9, 22.6 (cyclohexane-C), 46.9, 35.7, 32.4, 24.0 (tert-butyl carbons), $26.4\left(\mathrm{CH}_{3}\right)$; MS: $\mathrm{m} / z$ 470 [M $\mathrm{M}^{+}$; Anal. Calcd. for $\mathrm{C}_{23} \mathrm{H}_{30} \mathrm{~N}_{6} \mathrm{O}_{3} \mathrm{~S}$ (470.60): C, 58.70; H, 6.43; N, 17.86; Found: C, 58.55; H, 6.31; N, 17.95. 
3-Benzoyl-1-[4-(thiazol-2-yl-sulfonyl)phenyl]-1,2,4-triazaspiro[4.4]non-2-ene (6a): Yield: 66\%; m.p.: 219-221 ${ }^{\circ} \mathrm{C}$; IR (KBr) $v_{\max }: \mathrm{cm}^{-1} 3368,3345(\mathrm{NH}), 1675(\mathrm{Ph}-\mathrm{C}=\mathrm{O}), 1596(\mathrm{C}=\mathrm{N}), 1346,1175\left(\mathrm{SO}_{2}\right) ;{ }^{1} \mathrm{H}$ NMR (DMSO- $\left.d_{6}\right): \delta 11.56\left(s, 1 \mathrm{H}, \mathrm{SO}_{2} \mathrm{NH}\right), 8.27-7.06(\mathrm{~m}, 9 \mathrm{H}, \mathrm{Ar}-\mathrm{H}), 8.74(\mathrm{~d}, 1 \mathrm{H}$, thiazole $), 6.60(\mathrm{~d}, 1 \mathrm{H}$, thiazole), $5.63\left(\mathrm{~s}, 1 \mathrm{H}, \mathrm{NH}\right.$ triazole ring), 89.8 (spiro-C), 2.37-1.60 (m, 8H, cyclopentane); ${ }^{13} \mathrm{C}$ NMR (DMSO- $\left.d_{6}\right): \delta 185.2(\mathrm{C}=\mathrm{O}), 167.9,145.7,142.4,140.9,139.1,135.4,130.4,129.6,126.3,125.3,119.3$, 116.2 (Ar-C, $\mathrm{C}=\mathrm{N}$ and thiazole-C), 34.6, 24.7 (cyclopentane-C); MS: $m / z 467\left[\mathrm{M}^{+}\right.$]; Anal. Calcd. for $\mathrm{C}_{22} \mathrm{H}_{21} \mathrm{~N}_{5} \mathrm{O}_{3} \mathrm{~S}_{2}$ (467.57): C, 56.51; H, 4.53; N, 14.98; Found: C, 56.25; H, 4.40; N, 15.10 .

3-Benzoyl-1-[4-(pyrimidin-2-yl-sulfonyl)phenyl]-1,2,4-triazaspiro[4.4]non-2-ene (6b): Yield: 67\%; m.p.: 236-238 ${ }^{\circ} \mathrm{C}$; IR (KBr) $v_{\max }: \mathrm{cm}^{-1}$ 3371, 3347 (NH's), $1676(\mathrm{Ph}-\mathrm{C}=\mathrm{O}), 1597(\mathrm{C}=\mathrm{N}), 1346,1175\left(\mathrm{SO}_{2}\right) ;{ }^{1} \mathrm{H}$ NMR (DMSO-d $\left.d_{6}\right): \delta 11.58\left(s, 1 \mathrm{H}, \mathrm{SO}_{2} \mathrm{NH}\right), 8.27-7.06(m, 9 \mathrm{H}, \mathrm{Ar}-\mathrm{H}), 8.44(\mathrm{~d}, 2 \mathrm{H}$, pyrimidine), $6.81(\mathrm{t}$, $1 \mathrm{H}$, pyrimidine), $5.58\left(\mathrm{~s}, 1 \mathrm{H}, \mathrm{NH}\right.$ triazole ring), $2.37-1.60\left(\mathrm{~m}, 8 \mathrm{H}\right.$, cyclopentane); ${ }^{13} \mathrm{C}$ NMR (DMSO- $\left.d_{6}\right)$ : $\delta 185.5(\mathrm{C}=\mathrm{O}), 168.1,156.6,145.6,142.4,140.8,139.3,130.3,129.6,126.3,125.3,119.3,110.2(\mathrm{Ar}-\mathrm{C}$, $\mathrm{C}=\mathrm{N}$ and pyrimidine-C), 89.9 (spiro-C), 34.8, 24.7 (cyclopentane-C); MS: $\mathrm{m} / z 462\left[\mathrm{M}^{+}\right.$]; Anal. Calcd. for $\mathrm{C}_{23} \mathrm{H}_{22} \mathrm{~N}_{6} \mathrm{O}_{3} \mathrm{~S}$ (462.53): C, 59.73; H, 4.79; N, 18.17; Found: C, 59.55; H, 4.95; N, 18.05 .

3-(2-Furoyl)-1-[4-(thiazol-2-yl-sulfonyl)phenyl]-1,2,4-triazaspiro[4.4]non-2-ene (7a): Yield: 70\%; m.p.: 197-199 ${ }^{\circ} \mathrm{C}$; IR (KBr) v $v_{\max }: \mathrm{cm}^{-1} 3375,3355(\mathrm{NH} / \mathrm{s}), 1665(\mathrm{Ar}-\mathrm{C}=\mathrm{O}), 1616(\mathrm{C}=\mathrm{N}), 1346,1175\left(\mathrm{SO}_{2}\right) ;{ }^{1} \mathrm{H}$ NMR (DMSO- $\left.d_{6}\right): \delta 11.63\left(s, 1 \mathrm{H}, \mathrm{SO}_{2} \mathrm{NH}\right), 78.27-7.06(m, 7 \mathrm{H}, \mathrm{Ar}-\mathrm{H}), 8.66(\mathrm{~d}, 1 \mathrm{H}$, thiazole $), 6.58(\mathrm{~d}, 1 \mathrm{H}$, thiazole), $5.65\left(\mathrm{~s}, 1 \mathrm{H}, \mathrm{NH}\right.$ triazole ring), $2.37-1.60\left(\mathrm{~m}, 8 \mathrm{H}\right.$, cyclopentane); ${ }^{13} \mathrm{C}$ NMR (DMSO- $\left.d_{6}\right): \delta 173.6$ $(\mathrm{C}=\mathrm{O}), 168.2,142.2,139.6,135.6,138.8,129.8,129.5,128.2,127.4,125.6,119.3,116.4($ Ar- $\mathrm{C}, \mathrm{C}=\mathrm{N}$ and thiazole-C), 90.6 (spiro-C), 34.7, 24.6 (cyclopentane-C); MS: $m / z$ 457[ $\left.\mathrm{M}^{+}\right]$; Anal. Calcd. for $\mathrm{C}_{20} \mathrm{H}_{19} \mathrm{~N}_{5} \mathrm{O}_{4} \mathrm{~S}_{2}$ (457.53): C, 52.50; H, 4.19; N, 15.31; Found: C, 52.67; H, 4.06; N, 15.43 .

3-(2-Furoyl)-1-[4-(thiazol-2-yl-sulfonyl)phenyl]-1,2,4-triazaspiro[4.5]dec-2-ene (7c): Yield: 71\%; m.p.: 226-228 ${ }^{\circ} \mathrm{C}$; IR (KBr) $v_{\max }: \mathrm{cm}^{-1}$ 3377, $3352(\mathrm{NH}), 1666(\mathrm{Ar}-\mathrm{C}=\mathrm{O}), 1615(\mathrm{C}=\mathrm{N}), 1346,1175\left(\mathrm{SO}_{2}\right) ;{ }^{1} \mathrm{H}$ NMR (DMSO- $\left.d_{6}\right): \delta 11.64\left(s, 1 \mathrm{H}, \mathrm{SO}_{2} \mathrm{NH}\right), 8.27-7.06(m, 7 \mathrm{H}, \mathrm{Ar}-\mathrm{H}), 8.67(\mathrm{~d}, 1 \mathrm{H}$, thiazole $), 6.56(\mathrm{~d}, 1 \mathrm{H}$, thiazole), $\quad 5.60\left(\mathrm{~s}, 1 \mathrm{H}, \mathrm{NH}\right.$ triazole ring), $2.05-1.12\left(\mathrm{~m}, 10 \mathrm{H}\right.$, cyclohexane); ${ }^{13} \mathrm{C}$ NMR (DMSO- $\left.d_{6}\right): \delta$ $173.5(\mathrm{C}=\mathrm{O}), 168.2,142.3,139.7,135.7,138.8,129.8,129.2,128.2,127.3,125.6,119.4,116.5$ (Ar-C, $\mathrm{C}=\mathrm{N}$ and thiazole-C), 90.4 (spiro-C), 36.2, 25.1, 23.2 (cyclohexane-C); MS: $\mathrm{m} / z 471$ [M $\left.{ }^{+}\right]$; Anal. Calcd. for $\mathrm{C}_{21} \mathrm{H}_{21} \mathrm{~N}_{5} \mathrm{O}_{4} \mathrm{~S}_{2}$ (471.56): C, 53.49; H, 4.49; N, 14.85; Found: $\mathrm{C}, 53.35 ; \mathrm{H}, 4.55 ; \mathrm{N}, 14.73$.

3-(2-Thenoyl)-1-[4-(thiazol-2-yl-sulfonyl)phenyl]-1,2,4-triazaspiro[4.4]non-2-ene (8a): Yield: 72\%; m.p.: $218-220{ }^{\circ} \mathrm{C}$; IR (KBr) $v_{\max }: \mathrm{cm}^{-1} 3365,3345(\mathrm{NH}), 1665(\mathrm{Ar}-\mathrm{C}=\mathrm{O}), 1612(\mathrm{C}=\mathrm{N}), 1346,1175$ $\left(\mathrm{SO}_{2}\right) ;{ }^{1} \mathrm{H}$ NMR (DMSO- $\left.d_{6}\right): \delta 11.66,\left(\mathrm{~s}, 1 \mathrm{H}, \mathrm{SO}_{2} \mathrm{NH}\right), 8.27-7.06(m, 7 \mathrm{H}, \mathrm{Ar}-\mathrm{H}), 8.70(\mathrm{~d}, 1 \mathrm{H}$, thiazole), $6.63(\mathrm{~d}, 1 \mathrm{H}$, thiazole $), 5.63(\mathrm{~s}, 1 \mathrm{H}, \mathrm{NH}$ triazole ring $), 2.35-1.58\left(\mathrm{~m}, 8 \mathrm{H}\right.$, cyclopentane); ${ }^{13} \mathrm{C}$ NMR (DMSO- $\left.d_{6}\right): \delta 174.6(\mathrm{C}=\mathrm{O}), 168.1,145.4,142.2,141.8,135.6,134.8,129.4,128.0,127.8,125.7,120.8$,

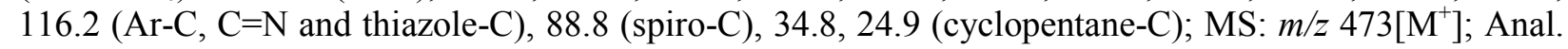
Calcd. for $\mathrm{C}_{20} \mathrm{H}_{19} \mathrm{~N}_{5} \mathrm{O}_{3} \mathrm{~S}_{2}$ (473.60): C, 50.72; H, 4.04; N, 14.79; Found: C, 50.90; H, 3.90; N, 14.65 .

3-(2-Thenoyl)-1-[4-(pyrimidin-2-yl-sulfonyl)phenyl]-1,2,4-triazaspiro[4.5]-dec-2-ene (8d): Yield: 73\%; m.p.: 198-200 ${ }^{\circ} \mathrm{C}$; IR (KBr) $v_{\max }: \mathrm{cm}^{-1} 3365,3346(\mathrm{NH}), 1660(\mathrm{Ar}-\mathrm{C}=\mathrm{O}), 1610(\mathrm{C}=\mathrm{N}), 1346,1175$ $\left(\mathrm{SO}_{2}\right) ;{ }^{1} \mathrm{H}$ NMR (DMSO- $\left.d_{6}\right): \delta 11.59\left(s, 1 \mathrm{H}, \mathrm{SO}_{2} \mathrm{NH}\right), 8.27-7.06(m, 7 \mathrm{H}, \mathrm{Ar}-\mathrm{H}), 8.38$ (d, 2H, pyrimidine), $6.81\left(\mathrm{t}, 1 \mathrm{H}\right.$, pyrimidine), $5.61\left(\mathrm{~s}, 1 \mathrm{H}, \mathrm{NH}\right.$ triazole ring), $2.04-1.13\left(\mathrm{~m}, 10 \mathrm{H}\right.$, cyclohexane); ${ }^{13} \mathrm{C}$ NMR $\left(\mathrm{DMSO}-d_{6}\right): \delta 174.8(\mathrm{C}=\mathrm{O}), 168.4,156.8,145.6,142.2,141.9,135.7,129.5,129.3,128.5,125.2,116.2$, 110.5 (Ar-C, C=N and pyrimidine-C), 88.9 (spiro-C), 36.1, 25.3, 23.1 (cyclohexane-C); MS: $\mathrm{m} / \mathrm{z} 482$ [M $]$; Anal. Calcd. for $\mathrm{C}_{22} \mathrm{H}_{22} \mathrm{~N}_{6} \mathrm{O}_{3} \mathrm{~S}_{2}$ (482.59): C, 54.76; H, 4.60; N, 17.41; Found: C, 53.60; H, 4.05; N, 19.35 .

3-(2-Naphthoyl)-1-[4-(thiazol-2-yl-sulfonyl)phenyl]-1,2,4-triazaspiro[4.4]non-2-ene (9a): Yield: 61\%; m.p.: $212-213^{\circ} \mathrm{C}$; IR $(\mathrm{KBr}) v_{\max }: \mathrm{cm}^{-1} 3369,3350(\mathrm{NH}), 1606(\mathrm{Ar}-\mathrm{C}=\mathrm{O}), 1608(\mathrm{C}=\mathrm{N}), 1346,1175\left(\mathrm{SO}_{2}\right)$; ${ }^{1} \mathrm{H}$ NMR $\left(\mathrm{DMSO}-d_{6}\right): \delta 11.66\left(s, 1 \mathrm{H}, \mathrm{SO}_{2} \mathrm{NH}\right), 8.27-7.06(m, 11 \mathrm{H}, \mathrm{Ar}-\mathrm{H}), 8.76(\mathrm{~d}, 1 \mathrm{H}$, thiazole $), 6.66(\mathrm{~d}$, $1 \mathrm{H}$, thiazole), 5.53 (s, $1 \mathrm{H}, \mathrm{NH}$ triazole ring), $2.37-1.60\left(\mathrm{~m}, 8 \mathrm{H}\right.$, cyclopentane); ${ }^{13} \mathrm{C}$ NMR (DMSO- $\left.d_{6}\right): \delta$ $185.4(\mathrm{C}=\mathrm{O}), 168.0,145.6,142.7,141.6,135.6,135.7,132.3,133.0,132.7,130.0,128.9,128.5,127.9$, $127.7,126.6,125.6,120.9,116.2$ (Ar-C, $\mathrm{C}=\mathrm{N}$ and thiazole-C), 89.8 (spiro-C), 34.9, 24.9 (cyclopentane- 
C); MS: $m / z 517$ [M $]$; Anal. Calcd. for $\mathrm{C}_{26} \mathrm{H}_{23} \mathrm{~N}_{5} \mathrm{O}_{3} \mathrm{~S}_{2}$ (517.63): C, 60.33; H, 4.48; N, 13.53; Found: C, $60.15 ; \mathrm{H}, 4.60 ; \mathrm{N}, 13.65$.

3-(2-Naphthoyl)-1-[4-(pyrimidin-2-yl-sulfonyl)phenyl]-1,2,4-triazaspiro[4.4]-non-2-ene (9b): Yield: 62\%; m.p.: 165-167 ${ }^{\circ} \mathrm{C}$; IR (KBr) $v_{\max }: \mathrm{cm}^{-1} 3367,3355$ (NH's), 1655 ( $\left.\mathrm{Ar}-\mathrm{C}=\mathrm{O}\right), 1605(\mathrm{C}=\mathrm{N}), 1346,1175$ $\left(\mathrm{SO}_{2}\right) ;{ }^{1} \mathrm{H}$ NMR $\left(\mathrm{DMSO}-d_{6}\right): \delta 11.67\left(s, 1 \mathrm{H}, \mathrm{SO}_{2} \mathrm{NH}\right), 8.27-7.06(m, 11 \mathrm{H}, \mathrm{Ar}-\mathrm{H}), 8.46(\mathrm{~d}, 2 \mathrm{H}$, pyrimidine), $6.85(\mathrm{t}, 1 \mathrm{H}$, pyrimidine $), 5.55(\mathrm{~s}, 1 \mathrm{H}, \mathrm{NH}$ triazole ring $), 2.34-1.51\left(\mathrm{~m}, 8 \mathrm{H}\right.$, cyclopentane) ${ }^{13} \mathrm{C}$ NMR (DMSO- $\left.d_{6}\right): \delta 185.6(\mathrm{C}=\mathrm{O}), 168.2,156.6,145.6,142.7,141.6,135.6,132.3,133.2,132.7,130.1$, 128.9, 128.5, 127.9, 127.6, 126.8, 125.7, 120.9, 110.4 (Ar-C, C=N and pyrimidine-C), 89.7 (spiro-C), 34.8, 24.7 (cyclopentane-C); MS: $m / z 512\left[\mathrm{M}^{+}\right]$; Anal. Calcd. for $\mathrm{C}_{27} \mathrm{H}_{24} \mathrm{~N}_{6} \mathrm{O}_{3} \mathrm{~S}$ (512.59): C, 63.27; $\mathrm{H}$, 4.72; N, 16.40; Found: C, 63.40; H, 4.62; N, 16.28 .

\section{Results and Discussion}

The precursors of amidrazones hydrazonoyl halides $\mathbf{1}$ employed, in this study, were prepared according to the reported literature procedures ${ }^{19-22}$. Treatment of the hydrazonoyl halides $\mathbf{1}$ with sodium azide in the presence of tetrabutylammonium iodide at room temperature gave azidohydrazones $\mathbf{2}$ (Figure 1). The obtained hydrazonoyl azides $\mathbf{2}$ were reacted with triphenylphosphine to afford phosphonimines $\mathbf{3}$ (Figure 1). It was found that the hydrolysis of compounds $\mathbf{3}$ with aqueous hydrochloric acid gave triphenylphosphine oxide and amidrazones 4 (Figure 1). A plausible mechanism for this acid hydrolysis could be an initial protonation of nitrogen followed by an attack from oxygen on the phosphorous atom ${ }^{19}$.

The condensation of amidrazones 4 with cyclic ketones in refluxing dioxane in the presence of catalytic amount of $p$-toluenesulfonic acid (PTSA) produced spiro 4,5-dihydro-1H-1,2,4-triazole derivatives containing sulfonamide moiety 5-9a-j (Figure 1) in good yields. The reaction progress was monitored by TLC to follow the completion of the synthesis.

The structures of the newly synthesized amidrazones 4 were elucidated on the basis of their spectroscopic data and elemental analyses. Electron impact (EI) mass spectra of the compounds 4a-j displayed the correct molecular ions $\left(\mathrm{M}^{+}\right)$in accordance with the suggested structures. The IR spectra exhibited typical stretching absorption bands of $\mathrm{C}=\mathrm{O}$ of conjugate ketone, anilide and ester groups at about $1725-1650 \mathrm{~cm}^{-1}$, NHs were observed in the region of $3470-3220 \mathrm{~cm}^{-1}$ and $1150,1060 \mathrm{~cm}^{-1}$ was attributed to $\mathrm{SO}_{2}$ of sulfonamide group. The ${ }^{1} \mathrm{H}$ NMR spectra of these amidrazones in DMSO- $d_{6}$ showed three characteristic signals, i.e. singlet at 12.6-12.4 ppm $\left(\mathrm{SO}_{2} \mathrm{NH}\right)$, singlet of the $\mathrm{NNH}$ group in the region of 6.3$6.0 \mathrm{ppm}$ and singlet of $\mathrm{NH}_{2}$ near 4.9-5.0 ppm. In addition, the characteristic singlet signal of the CONH group in compounds containing anilide group was observed at about 10.8-9.8 ppm and the expected proton signals of the aromatic rings were in the range of 8.4-6.9 ppm. The ${ }^{13} \mathrm{C}$ NMR spectra exhibited characteristic signals for the $\mathrm{Ar}-\mathrm{C}=\mathrm{O}$ carbon at about $192-159 \mathrm{ppm}$ and for the $\mathrm{C}=\mathrm{N}$ moiety at about $143-141 \mathrm{ppm}$.

The structural assignment of the prepared spiro 1,2,4-triazoles 5-9a-j was based on elemental analysis, spectral data and literature values ${ }^{20-22}$. Physical properties, molecular ion peaks and microanalysis are presented in experimental section. The IR spectra of these compounds revealed the presence of NH band of dihydrotriazole ring resonating near $3370-3350 \mathrm{~cm}^{-1}$. In addition to the bands of characteristic functional groups, the ${ }^{1} \mathrm{H}$ NMR spectra of products 5-9a-j in DMSO- $d_{6}$ display a characteristic singlet in the region of 5.6-5.5 ppm due to $\mathrm{NH}$ proton of dihydrotriazole ring. Also, the spectra exhibited a characteristic singlet at 12.7-12.6 ppm due to $\mathrm{SO}_{2} \mathrm{NH}$ proton. The ${ }^{13} \mathrm{C}$ NMR spectra displayed characteristic signals of the suggested structures. The signal at $90-95 \mathrm{ppm}$, which is attributed to C-5 (spiro carbon) of dihydrotriazole ring was of special significance, which is similar to the reported values of spiro carbons flanked by two nitrogens in five-membered heterocycles ${ }^{23,25}$. The spectral data of the obtained compounds 5-9a-j are summarized in the experimental section. 


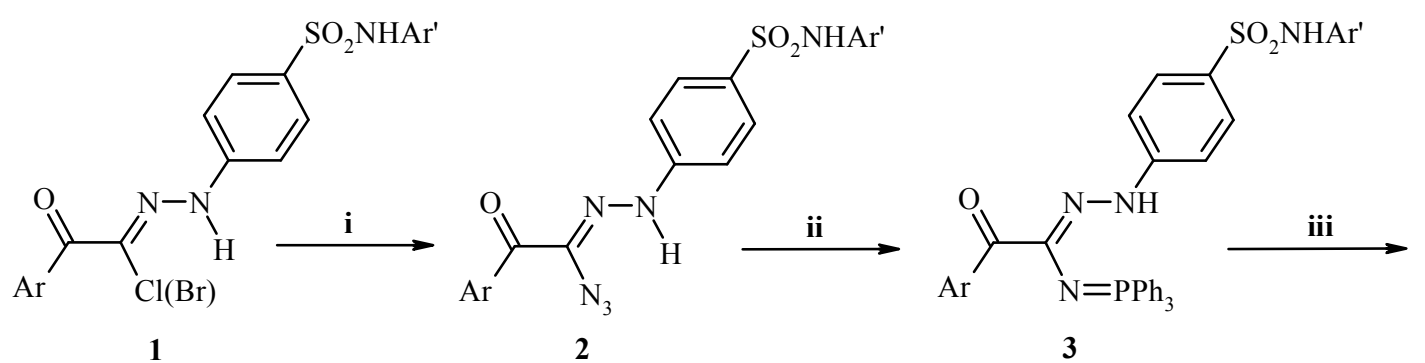

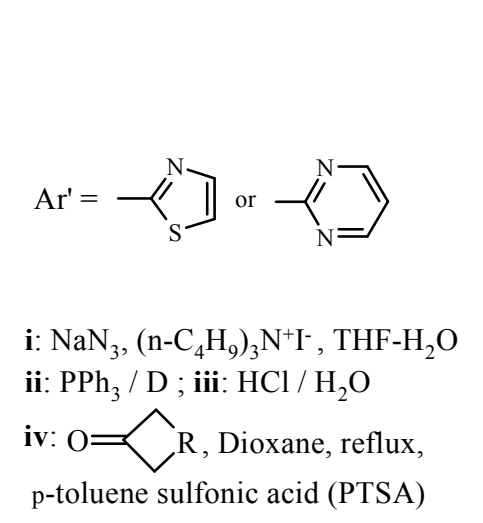

$$
\begin{aligned}
& \quad \mathrm{Ar} / \mathrm{R} \\
& \text { a, 2-thiazlyl } /\left(\mathrm{CH}_{2}\right)_{2} ; \\
& \text { b, 2-pyrimidinyl } /\left(\mathrm{CH}_{2}\right)_{2} \text {; } \\
& \text { c, 2-thiazolyl } /\left(\mathrm{CH}_{2}\right)_{3} ; \\
& \text { d, 2-pyrimidinyl } /\left(\mathrm{CH}_{2}\right)_{3} \text {; } \\
& \text { e, 2-thiazolyl } /\left(\mathrm{CH}_{2}\right)_{4} ;
\end{aligned}
$$

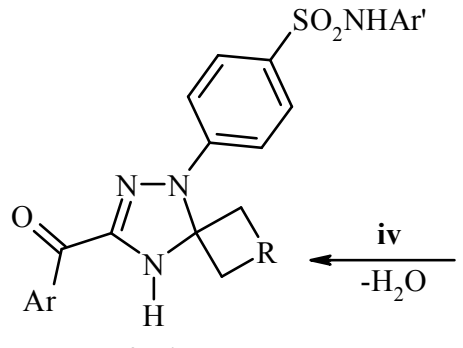

5-9a-j

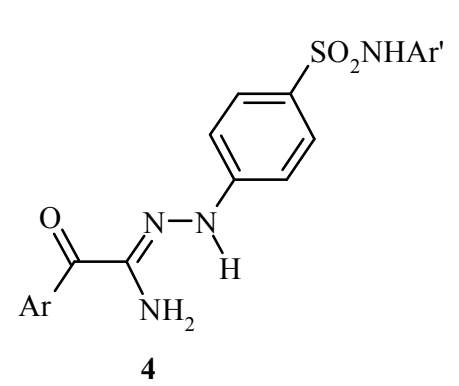

5: $\mathrm{Ar}=\mathrm{Me} ; \mathbf{6}: \mathrm{Ar}=\mathrm{Ph} ; \mathbf{7}: \mathrm{Ar}=2-\mathrm{Furyl}$;

8: $\mathrm{Ar}=2$-Theinyl; 9: $\mathrm{Ar}=2$-Naphthyl

$$
\mathrm{Ar}^{\prime} / \mathrm{R}
$$

f, 2-pyrimidinyl $/\left(\mathrm{CH}_{2}\right)_{5}$;

\begin{tabular}{|c|c|c|c|c|c|c|c|}
\hline \multicolumn{8}{|c|}{ Diameter of the inhibition zone in $\mathrm{mm}^{*}$} \\
\hline \multirow{2}{*}{$\begin{array}{l}\text { Cpd. } \\
\text { No. }\end{array}$} & \multicolumn{5}{|c|}{ Antibacterial activity } & \multicolumn{2}{|c|}{ Antifungal activity } \\
\hline & Euterococci & E. coli & S. aureus & Klebsiella spp & Proteus spp & C. albicans & A. niger \\
\hline 5a & 16 & 17 & 17 & 13 & 16 & 15 & 14 \\
\hline $5 \mathbf{b}$ & 13 & 18 & 15 & 11 & 10 & 17 & 18 \\
\hline $5 \mathrm{c}$ & 19 & 15 & 11 & 14 & 16 & 18 & 16 \\
\hline 5d & 18 & 16 & 17 & 18 & 19 & 16 & 12 \\
\hline $6 \mathbf{b}$ & 16 & 19 & 16 & 19 & 11 & 19 & 11 \\
\hline $7 \mathbf{a}$ & 13 & 12 & 14 & 16 & 17 & 16 & 19 \\
\hline $7 b$ & 19 & 15 & 11 & 14 & 16 & 18 & 16 \\
\hline $\mathbf{8 a}$ & 18 & 16 & 17 & 18 & 19 & 16 & 12 \\
\hline $8 b$ & 16 & 19 & 16 & 19 & 11 & 19 & 11 \\
\hline $9 a$ & 13 & 12 & 14 & 16 & 17 & 16 & 19 \\
\hline Tet. $^{\mathrm{a}}$ & 21 & 20 & 19 & 21 & 22 & & \\
\hline Flu. $^{b}$ & - & - & - & - & - & 21 & 23 \\
\hline DMF & - & - & - & - & - & - & - \\
\hline
\end{tabular}

g, 2-thiazolyl/-- $\mathrm{CH}_{2} \mathrm{CHMeCH}_{2}$-;

h, 2-pyrimidinyl/ $-\mathrm{CH}_{2} \mathrm{CHMeCH}_{2}$;

i, 2-thiazolyl/- $\mathrm{CH}_{2} \mathrm{CH}(\mathrm{t}-\mathrm{Bu}) \mathrm{CH}_{2}$-;

j, 2-pyrimidinyl/- $\mathrm{CH}_{2} \mathrm{CH}(\mathrm{t}-\mathrm{Bu}) \mathrm{CH}_{2}-$

Figure 1. Synthetic pathway for the preparation of compounds 4 and 5-9a-j.

Table 1. Antimicrobial screening results of the tested compounds

${ }^{*}$ Calculated as average of three values. ${ }^{\mathbf{a}}$ Tetracycline, ${ }^{\mathbf{b}}$ Fluconazole 
A standard nutrient agar disc diffusion method ${ }^{26,27}$ was followed to determine the activity of the synthesized compounds against the sensitive organisms Euterococci, Escherichia coli, Staphylococcus aureus, Klebsiella spp and Proteus spp, as bacterial strains and two species of fungi, namely Aspergillus niger and Candida albicans. The compounds were tested at a concentration of $1 \mathrm{mg} \mathrm{mL}^{-1}$ in $N, N$-dimethyl formamide (DMF) solution, measuring the average diameter of the inhibition zone in $\mathrm{mm}$. The results showed that all the tested compounds exhibited a marked degree of activity against bacteria and fungi compared with well-known antibacterial and antifungal substances such as tetracycline and fluconazole. According to $\mathrm{NCCLS}^{27}$, inhibition zones for tetracycline and fluconazole $<14 \mathrm{~mm}$ is considered resistant, between 15 and $18 \mathrm{~mm}$ is considered weakly sensitive and $>19 \mathrm{~mm}$ is considered sensitive. Also, the results showed that the degree of inhibition varied with the tested compounds (Table 1). The thiazolyl and pyrimidinyl moieties generally led to a dramatic improvements in activity against both bacteria and fungi. The present study can lead medicinal chemists to design and synthesize similar compounds with enhanced biological potency in future.

\section{Conclusion}

New series of novel functionalized spiro1,2,4-triazols 5-9a-j bearing sulfonamide moiety were synthesized upon treatment of amidrazones 4a-j with cyclic ketones in refluxing dioxane, which were examined for their antimicrobial activities, and they were found to possess various antimicrobial activities towards all the microorganisms tested. Shortly, the biological activity is strongly dependent on the nature and size of the substituents on triazole ring.

\section{Acknowledgements}

The authors are great thankful to the Qatar Charity for the financial support of this research through Ibhath grant (GCC-07-06).

\section{ORCID}

Hany M. Dalloul: 0000-0002-4947-3648

Khaled El-nwairy: 0000-0001-7824-9680

Ali Z. Shorafa: 0000-0001-9906-5389

Ahmed Abu Samaha: 0000-0001-7705-6086

\section{References}

[1] Kuhn, D. G.; Furch, J. A.; Hunt, D. A.; Asselin,M.; Baffic, S. P.; Diehl, R. E.; Miller, T. P.; Palmer, Y. L.; Treacy, M. F.; Trotto, S. H. Cycloalkyl-substituted amidrazones: A novel class of insect control agents. ACS. Symp. Ser. 1998, 686, 185-193.

[2] Neilson, D. G.; Roger, R.; Heatlie, J. W. M.; Newlands, L. R. Chemistry of amidrazones Chem. Rev. 1970, $70,151-170$.

[3] Aly, A. A.; Nour-El-Din, A. M. Functionality of amidines and amidrazones Arkivoc 2008, (i), 153-194.

[4] Mamolo, M. G.; Vio, L.; Banfi, E.; Predominato, M.; Fabris, C.; Asaro, F. Synthesis and antimyco-bacterial activity of some 2-pyridinecarboxyamidrazone derivatives. IL Farmaco 1992, 47, 1055-1066.

[5] Mamolo, M. G.; Vio, L.; Banfi, E.; Predominato, M.; Fabris, C.; Asaro, F. Synthesis and antimyco-bacterial activity of some 4-pyridinecarboxyamidrazone derivatives. IL Farmaco 1993, 48, 529-538.

[6] Ranft, D.; Seyfarth, T.; Schaper, K. J.; Lehwark-Yvetot, G.; Bruhn, C.; Buge, A. New N¹-Hetaryl-methylenesubstituted amidrazones with potential antimycobacterial activity. Arch. Pharm. Pharm. Med. Chem. 1992, $332,427-430$.

[7] Modzelewska-Banachiewic, B.; Matysiak, J.; Niewiadomy, A. Synthesis and mycological activity of the compounds obtained in the reaction of $N^{3}$-substituted amidrazones with sulphinyl-bis-2,4-dihydroxybenzenethioyl. Eur. J. Med. Chem. 2001, 36, 75-80.

[8] Abdel-Jalil, R.; J.; Al Momani, E.; Hamad, M.; Voelter, W.; Mubarak, M. S.; Smith, B. H.; Peters, D. G. Synthesis, antitumor activity andelectrochemical behavior of some piperazinyl amidrazones. Monatsh. Chem. 2010, 141, 251-258.

[9] Dean, G. S.; Rhodes, S. G.; Coad, M.; Whelan, A. O.; Wheeler, P.; Villareal-Ramos, B.; Mead, E.; Johnson, L.; Clifford, D. J.; Hewinson, R. G.; Vordermeier, H. M. Isoniazid treatment of Mycobacterium bovis in cattle as a model for human tuberculosis. Tuberculosis 2008, 88, 586-594. 
[10] Hackmann, J. T. US Patent 2758 050, 1956; Haldeman, R. G.; Morin, L. T.; Matsuda, K. US Patent 3 073013, 1963.

[11] Neilson, D. G.; Roger, J. W.; Heatlie, L. R. Chemistry of amidrazones Chem. Rev. 1970, 70, 151-170.

[12] Dalloul, H. M.; Boyle, P. H. Synthesis of nitrogen-containing heterocycles from the reaction of amidrazones with $\alpha$-haloesters. Turkish J. Chem. 2007, 31, 141-147.

[13] Borad, M.A.; Bhoia, N.; Prajapatia, N. P.; Patela, H. D. Review of synthesis of spiro heterocyclic compounds from Isatin. Synth. Commun. 2013, 44(7), 897-922.

[14] Shawali, A. S.; Abdelhamid, A. O. Synthesis of Spiro-heterocycles via 1,3-dipolar cycloadditions of nitrilimines to exoheterocyclic enones. site-, regio- and stereo-selectivities overview . Curr.Org. Chem. 2012, 16, 2673-2689.

[15] Dalloul, H. M.; Synthesis of nitrogen-containing dispiroheterocyles (I) using nitrilimines. Arkivoc, 2008 (xiv) 234-241.

[16] Dalloul, H. M.; Abu samaha, A.; Synthesis of nitrogen-containing dispiroheterocyles (II) using nitril-imines. J. Serb. Chem. Soc., 2010, 75, 1473-1479.

[17] Dalloul, H. M. Synthesis of new spiro-heterocycles containing dihydrotetrazine moiety South. Braz. J. Chem. Soc. 2014, 22, 17-26.

[18] Mok, B. L. PhD, The Synthesis of Functionalized Sulfonamides Chemistry Department, University College London, UK, 2008.

[19] Shawali, A. S.; Hassaneen, H. M.; Fahmy, A. A.; Abunada, N. M.; Mousa, H. A. Synthesis and reactions of triphenylphosphoniminoglyoxalic acid anilide arylhydrazones. Phosphor. Sulfur Sil. 1990 53, 259-265.

[20] Ashok, D.; Ganesh, A.; Lakshmi, V.B.; Ravi, S.; Ramesh, B. Microwave assisted synthesis and antimicrobial activity of novel 1-[1/2-(1-Benzyl-1H-[1,2,3]triazol-4-ylmethoxy)-naphthalen-2/1-yl]-3-(1-phenyl-3-aryl-1Hpyrazol-4-yl)-propenones. Org. Commun. 2014, 8, 24-35.

[21] Sreedhar, B.; Reddy, T.V., Raju, C.N., Reddy, G.V.S. Design, synthesis, characterization and bioassay of novel carboxamide derivatives of Celecoxib. Org. Commun. 2016, 9, 54-64.

[22] Drutkowski, G.; Donner, C.; Schulze, I.; Frohberg, P. Novel method for the synthesis of 1,2,4-triazoles and 1,2,4-triazol-3-ones. Tetrahedron, 2002, 58, 5317-5326.

[23] Ferwanah, A.S.; Awadallah, A.M.; El-Sawi, E.A. Dalloul, H.M. Synthesis of dihydro- and tetra-hydro-1,2,4,5tetrazines from the reaction of nitrilimines with 1-Substituted-1-methylhydrazine. Synth. Commun. 2003, 33, 1245-1253.

[24] Dalloul, H. M.; Boyle, P. H. Heterocyclic synthesis using nitrilimines: Part 3. Synthesis of substituted 1,2,4,5-tetrazines and 1,2,4,5-tetraaza-2-pentenes. Heterocycl. Commun. 2003, 9, 507-514.

[25] Dalloul, H. M.; Heterocyclic synthesis using nitrilimines: Part 4. Synthesis of 3-substituted 1-aryl-1,2,4triazaspiroalk-2-nes. Chem. Heterocycl. Comp. 2004, 40, 1402-1407.

[26] Manika, M.; Chanotiya, C.S.; Darokar, M.; Singh, S.; Bagchi, G.D. Compositional characters and antimicrobial potential of Artemisia stricta Edgew. f. stricta Pamp. essential oil. Rec. Nat. Prod. 2016, 10, 40-46

[27] Clinical and Laboratory Standards Institute, "Methods for dilution antimicrobial susceptibility tests for bacteria grow aerobically", Approved Standard M7-A4, Clinical and Laboratory Standards Institute, Wayne, Pa, USA, 2005.

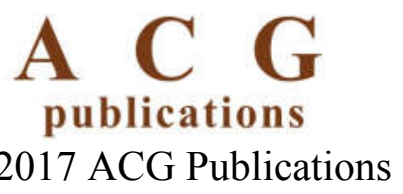

